\title{
Acute Compartment Syndrome in the Anabolic Steroid using Bodybuilder: A Case Report
}

\author{
Eduardo Freitas Ferreira*, Barbara Dantas, Diogo Portugal, Nuno Silva, Catarina \\ Peixoto, André Ladeira and Carla Vera-Cruz
}

Department of Physical Medicine and Rehabilitation, Hospital Professor Doutor Fernando Fonseca, Portugal

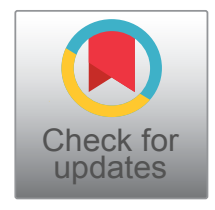

*Corresponding author: Eduardo Freitas Ferreira, Department of Physical Medicine and Rehabilitation, Hospital Prof. Doutor Fernando Fonseca E.P.E, IC 19-Venteira, 2720-276, Amadora, Portugal, Tel: 00351-214-348-200

\begin{abstract}
Anabolic steroids are frequently used by bodybuilders in order to achieve a rapid increase in muscle mass and strength. However, this practice may be associated with several complications including a compartment syndrome.

A 19-year-old male bodybuilder presented to the emergency department with a lower right leg anterior non-traumatic compartment syndrome associated with a footdrop. He had been using daily anabolic-androgenic steroids for the past 12 weeks. Emergency fasciotomy was done and the patient initiated rehabilitation.

Muscles undergoing rapid hypertrophy within a less elastic fascia, as the result of exercise and anabolic steroids, may be more susceptible to compartment syndromes. Increased intra-compartimental pressure originates myocyte necrosis and nerve injury. Thus, in order to preserve muscle and nerve integrity, an emergency fasciotomy in necessary. Steroid acquisition is done over the internet or the black market with little control or regulation. Hence, a greater awareness of this problem and acquisition control is mandatory.
\end{abstract}

\section{Keywords}

Athlete, Anabolic steroids, Bodybuilder, Compartment syndrome

\section{Introduction}

Bodybuilders frequently use anabolic steroids in order to achieve a rapid increase in muscle mass and strength. However, this practice may be associated with important complications. Although the frequency of compartment syndrome is increased in athletes due to excessive exercise, steroid usage accelerates the risk
[1]. Acute compartment syndrome is an emergency, requiring immediate fasciotomy in order to preserve muscle and nerve integrity [2]. However in non-traumatic compartment syndrome diagnosis is usually delayed originating worse functional prognosis [3].

Only a few case reports associating compartment syndrome with steroid use have been published and the majority in the traumatic setting. This report presents the case of a young bodybuilder using steroids presenting with a non-traumatic compartment syndrome.

\section{Case Report}

A 19-year-old male with no past medical history presented to the Emergency Department (ED) with a 24 hour evolution of right leg pain and swelling without a trauma history. The patient performed frequent sessions of resistance exercises focused on muscle hypertrophy. He had been taking a daily oral and injectable anabolic-androgenic steroids mixture (testosterone, trenbolone, winstrol, boldenone, clemetrol, oxandrolone and triiodothyronine) for the last 12 weeks. Injection sites included the upper limbs, buttocks and lower limbs. Examination revealed an edematous, slightly erythematosus and warm right leg with ankle dorsiflexion limitation. Laboratory findings demonstrated leucocytosis $(12000 / \mu \mathrm{l})$, an elevated creatinine kinase $(1981 \mathrm{U} / \mathrm{l})$ and a slight hepatic cytolysis pattern (AST $100 \mathrm{U} / \mathrm{I}$ and ALT $72 \mathrm{U} / \mathrm{I}$ ). D-dimer, electrolyte and renal analysis were normal. Leg radiography and Doppler studies failed to show any abnormalities. The pa-

Citation: Ferreira EF, Dantas B, Portugal D, Silva N, Peixoto C, et al. (2020) Acute Compartment Syndrome in the Anabolic Steroid using Bodybuilder: A Case Report. Int J Sports Exerc Med 6:181. doi. org/10.23937/2469-5718/1510181

Accepted: December 29, 2020; Published: December 31, 2020

Copyright: (C) 2020 Ferreira EF, et al. This is an open-access article distributed under the terms of the Creative Commons Attribution License, which permits unrestricted use, distribution, and reproduction in any medium, provided the original author and source are credited. 


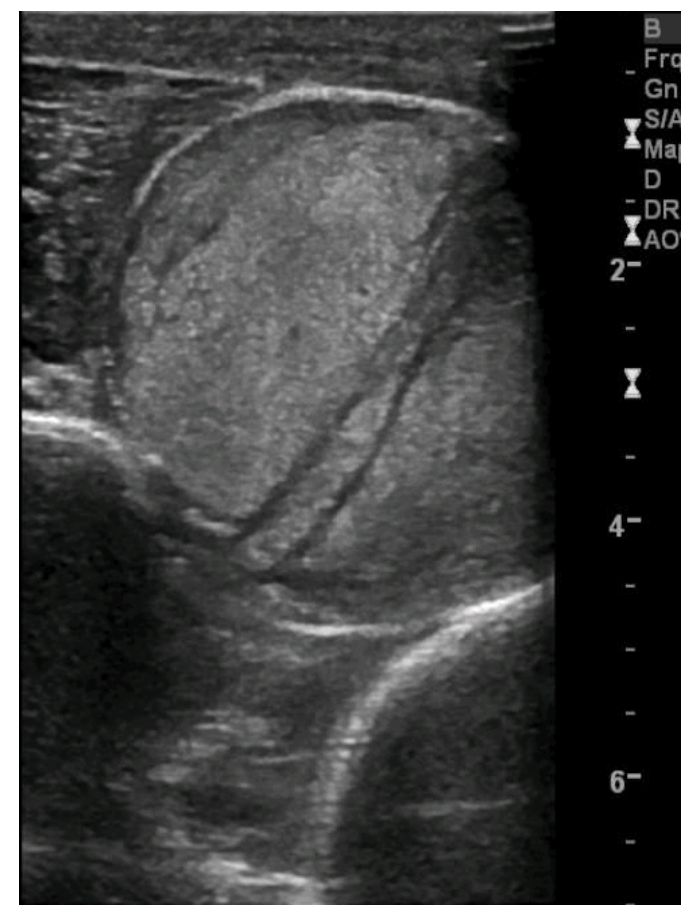

(A)

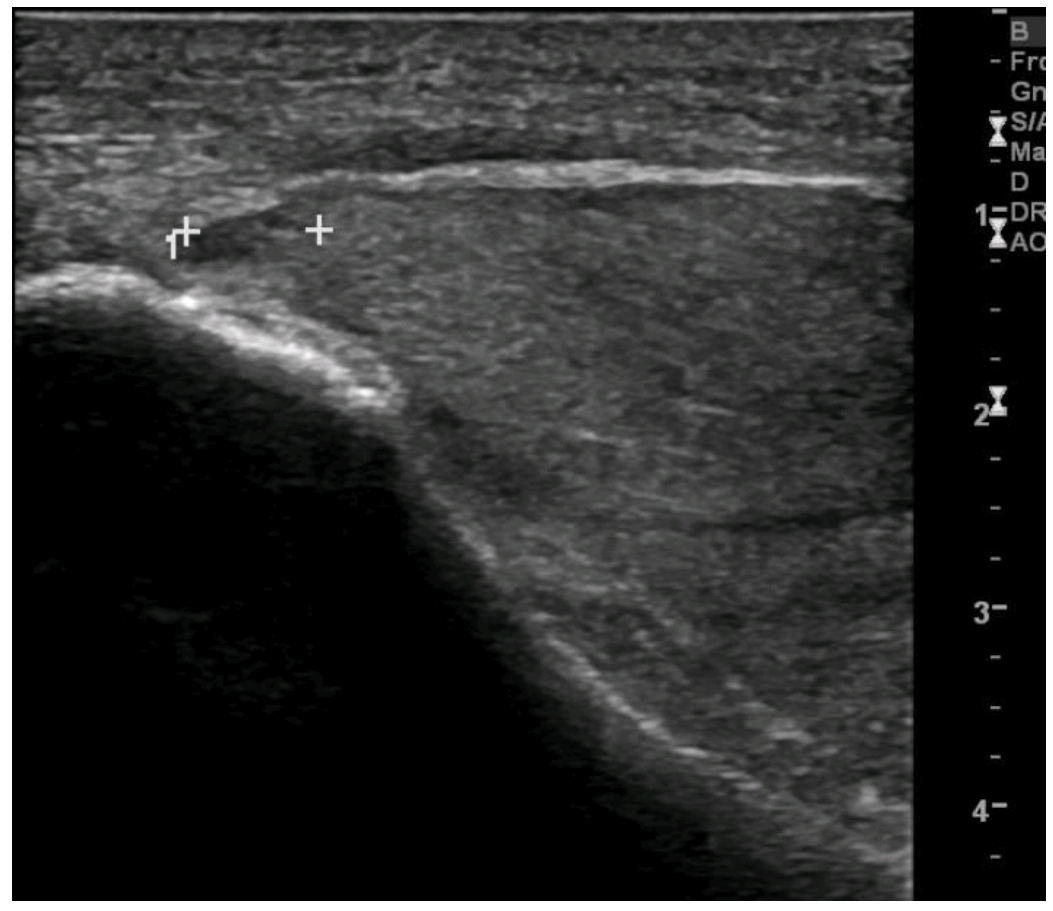

(B)

Figure 1: Right Leg ultrasonography (A) An increase muscle volume (tibialis anterior muscle) with a fibrillar pattern and diffuse hyperechogenicity, associated with slight oedema, reflecting an inflammatory process with a possible hematoma component; (B) Image compatible with proximal muscle rupture of $7 \mathrm{~mm}$.

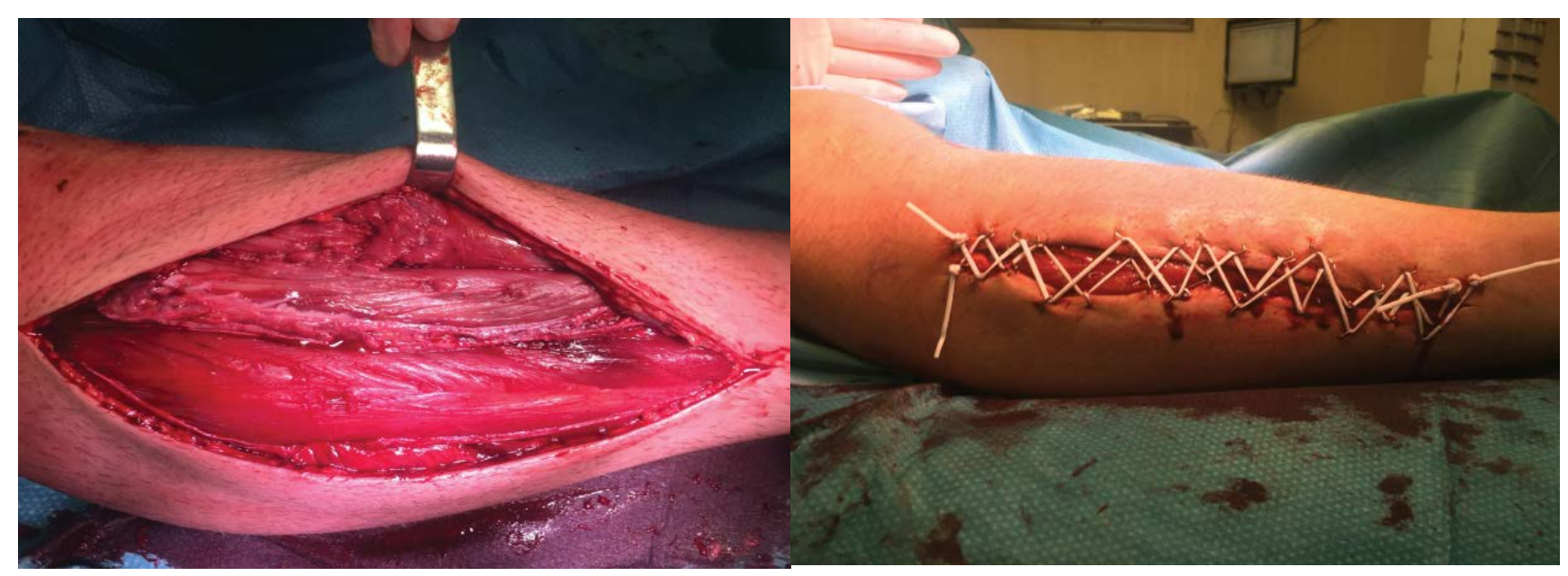

Figure 2: Right Leg Fasciotomy. Photographs courtesy of Dr. Mafalda Lopes.

tient was discharged from the ED with analgesia and indication to rest, cryotherapy and stop steroids.

After 10 days the patient returns to the ED with aggravating symptoms. Examination demonstrated tension and pain over the anterior compartment of the leg, hallux hypoesthesia and inability to dorsiflex his right ankle and extend the right hallux and toes. Laboratory findings showed a slight decrease in creatinine kinase (1347 U/I), maintaining slight leucocytosis $(11400 / \mu l)$ and elevation of the hepatic cytolysis pattern (AST 167 $\mathrm{U} / \mathrm{I}$ and ALT $264 \mathrm{U} / \mathrm{I}$ ). Leg ultrasonography showed no vascular abnormalities but revealed increase muscle volume (tibialis anterior) with a fibrillar pattern and diffuse hyperechogenicity, associated with slight oedema, reflecting an inflammatory process with a possible hematoma component (Figure $1 \mathrm{~A}$ and Figure 1B). The patient was diagnosed with an acute anterior compartment syndrome of the right leg associated with rhabdomyolysis and neurological suffering. Right leg pressure measurements revealed an anterior compartment pressure of $80 \mathrm{mmHg}$ whilst lateral and posterior compartments showed normal values. On this basis, an emergent anterolateral right leg fasciotomy was performed (Figure 2). Intra-operative exploration revealed a devitalized tibialis anterior muscle but an intact peroneal nerve. Fasciotomy closure was done 5 days later. 

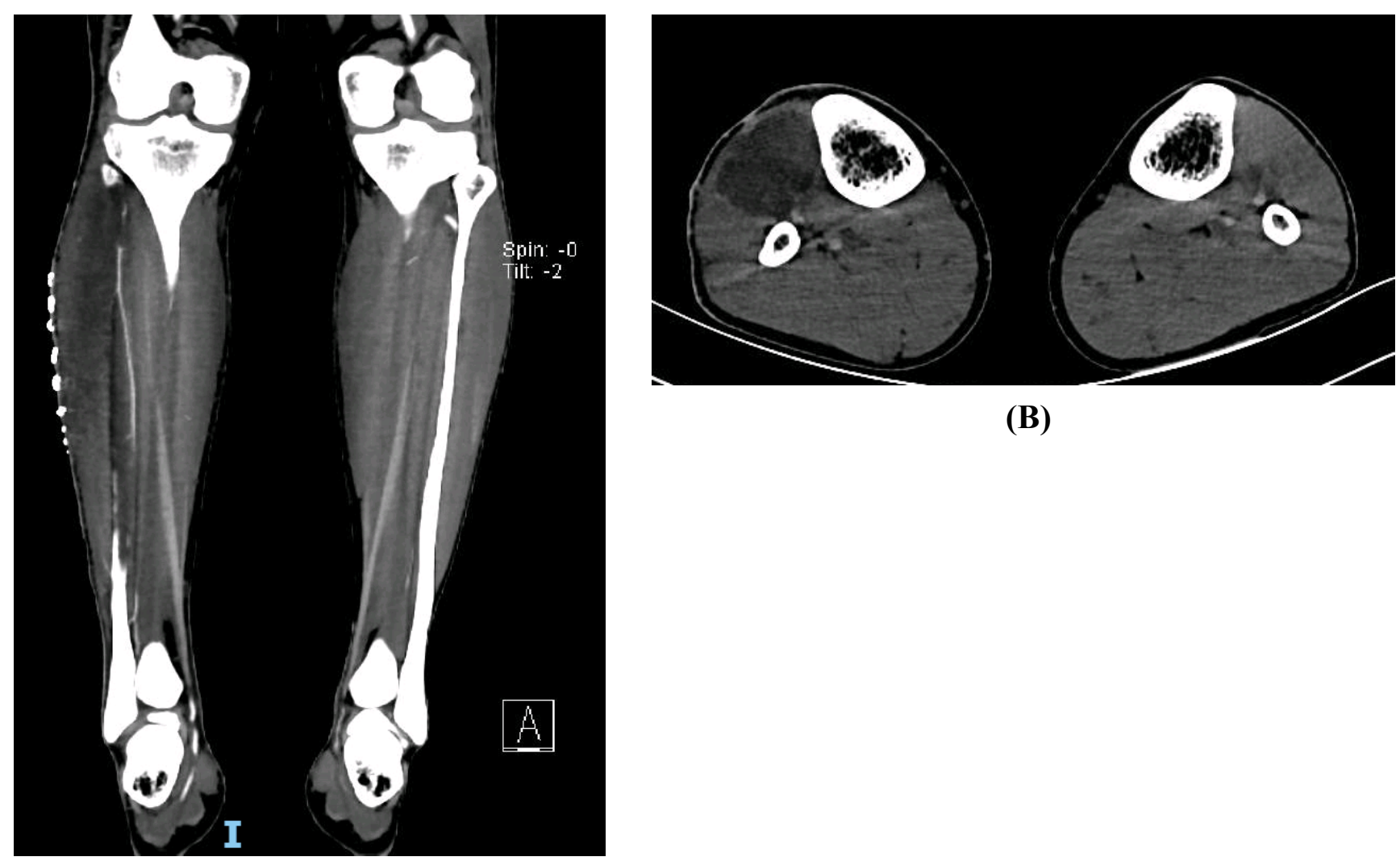

(B)

(A)

Figure 3: Leg TC Scan. A large expansive heterogeneous area in the muscle plane of the external aspect of the right leg, encompassing the muscles and tendons of tibialis anterior and long extensors of the fingers and hallux, related to evolving muscle necrosis, consequent to the compartment syndrome (A) Coronal plane; (B) Transverse plane.

During hospitalization the patient maintained inability to dorsiflex his right ankle and extend hallux and toes. A rehabilitation program was initiated consisting of lower extremity muscle strengthening, articular mobilization and gait training. Due to right footdrop interfering with walking, an Ankle-Foot Orthosis (AFO) (Boxia ${ }^{\circ}$ ) was prescribed. Control CT scan revealed a large expansive heterogeneous area in the muscle plane of the external aspect of the right leg, encompassing the muscles and tendons of tibialis anterior, extensor hallucis longus and extensor digitorum longus, related to muscle necrosis, consequent to the compartment syndrome (Figure $3 \mathrm{~A}$ and Figure 3B).

The patient was revaluated by physical medicine and rehabilitation one month after hospital discharge. On examination he maintained footdrop, although walking was possible without aids with a compensated gait (greater knee flexion). However patient refused to use the prescribed AFO. Electrodiagnostic studies demonstrated right severe axonal deep peroneal nerve injury with no muscle activity in the tibialis anterior muscle. The patient continued rehabilitation program in a community clinic. However, on revaluation, he maintained right footdrop even after rehabilitation and returned to his bodybuilding training but refrained from using steroids. To restore ankle lost function, the orthopedic department proposed tendon transfers or arthrodesis that the patient refused.

\section{Discussion}

Anabolic-androgenic steroid injection is frequently used by bodybuilders [1]. In the majority of cases, in countries were steroid selling is regulated, acquisition is done over the black market with little control or regulation. The main reason for steroid usage is to enhance muscle mass and strength. However, anabolic steroids have been linked to several complications including neuromuscular complications such as mononeuropathies [4] and compartment syndromes [1]. Muscles undergoing rapid hypertrophy within a less elastic fascia, as the result of exercise and anabolic steroids use, may be more susceptible to compartment syndrome $[1,4]$. It is postulated that steroids have metabolic effects that render the muscle less tolerant to compartment hypertension and ischemia [5]. Additionally, steroid injections may also cause localized rhabdomyolysis in the injection site contributing to compartment syndrome development [6].

Compartment syndromes of the lower extremity are characterized by an increased pressure in a tight fascial compartment [7]. As intra-compartimental pressure rises above blood pressure, arterial flow to the muscle is reduced originating myocyte ischemia and muscle necrosis [7]. Furthermore, diminished lymphatic and venous return leads to fluid and inflammatory mediator accumulation which further increase compartment 
pressure [8]. Although traumatic events represent the vast majority of cases [9], non-traumatic causes like excessive exercise and steroid usage must also be considered, especially in athletes.

In the lower extremity the anterior compartment of the leg is the most frequently affected site [10]. It contains the tibialis anterior, extensor hallucis longus and extensor digitorum longus muscles, the anterior tibial artery and the deep peroneal nerve. Thus, increased intra-compartmental pressure may cause nerve injury, developing a footdrop.

Diagnosis of non-traumatic compartment syndrome is frequently delayed due to its uncommonness [3]. Thus, a high suspect index is necessary in order to reduce muscle necrosis and functional impairment. Emergency fasciotomy is necessary when intra-compartimental pressure exceeds $30 \mathrm{mmHg}$ [9]. However, faster damage occurs at higher pressures [5] or more prolonged times [11]. Studies suggest that permanent disability is inevitable if fasciotomies are delayed more than 12 hours [11].

Peroneal neuropathy may originate gait impairment. If the patient has a subtle injury, strengthening may help functional recovery, however, if a severe injury is present or if associated with major muscle necrosis, passive range of motion in order to prevent contractures may be all that is possible [12]. In this setting, an AFO may be a valuable aid in order to facilitate walking and correct gait pattern and surgical solutions such as tendon transfers or ankle arthrodesis may be considered.

This paper presents the case of a young bodybuilder using steroids presenting to the ED with a non-traumatic compartment syndrome. Although steroids are commonly used by these athletes with little control, they may be associated with severe complications. Thus, a greater awareness of this problem and acquisition control is mandatory.

\section{Acknowledgments}

The authors would like to acknowledge Dr. Mafalda Lopes for providing intraoperative fasciotomy photographs.

\section{Funding Disclosure}

The authors report no conflicts of interest.

\section{Authors Declaration}

Eduardo Freitas Ferreira, Bárbara Dantas, Diogo Portugal, Nuno Silva, Catarina Peixoto, André Ladeira and Carla Vera-Cruz contributed equally in the work conception, acquisition and interpretation of data, drafting and revising, final approval of the version for publishing and agreement to be accountable for all aspects of the work.

\section{References}

1. Bahia H, Platt A, Baguley P (2000) Anabolic steroid accelerated multicompartment syndrome following trauma. $\mathrm{Br} \mathrm{J}$ Sports 34: 308-309.

2. Rowland SA (1993) Fasciotomy: the treatment of compartment syndrome. In: Green DP, Operative hand surgery. (3 ${ }^{\text {rd }}$ edn), Edinburgh: Churchill Livingstone, 661-694.

3. Livingston KS, Meehan WP, Hresko MT (2018) Acute exertional compartment syndrome in young athletes: A descriptive case series and review of the literature. Pediatr Emerg Care 34: 76-80.

4. Mondelli M, Cioni R, Federico A (1998) Rare mononeuropathies of the upper limb in bodybuilders. Muscle Nerve 21: 809-812.

5. Liem NR, Bourque PR, Michaud C (2005) Acute exertional compartment syndrome in the setting of anabolic steroids: an unusual cause of bilateral footdrop. Muscle Nerve 32: 113-117.

6. Farkash U, Shabshin N (2009) Rhabdomyolysis of the Deltoid Muscle in a Bodybuilder Using Anabolic-Androgenic Steroids: A Case Report. J Athl Train 44: 98-100.

7. Tzioupis C, Cox G, Giannoudis P (2009) Acute compartment syndrome of the lower extremity: An update. Orthop Trauma 13: 433-440.

8. Hutchinson MR, ML Ireland (1994) Common compartment syndromes in athletes. Treatment and rehabilitation. Sports Med 17: 200-208.

9. McLaughlin N, Heard H, Kelham S (2014) Acute and chronic compartment syndromes: Know when to act fast. JAAPA 27: $23-26$.

10. Dombek DM, Lamm BM, Saltrick K (2003) Peroneal tendon tears: a retrospective review. J Foot Ankle Surg 42: 250258.

11. Fehlandt A, Micheli L (1995) Acute exertional anterior compartment syndrome in an adolescent female. Med Sci Sports Exerc 27: 3-7.

12. Baima J, Krivickas L (2008) Evaluation and treatment of peroneal neuropathy. Curr Rev Musculoskelet Med 1: 147153. 